\title{
The Effects of Vortex Ingestion on the Flow in a Diffusing S-Duct
}

B. J. Wendt and B. A. Reichert Lewis Research Center Cleveland, Ohio

Prepared for the 30th Joint Propulsion Conference cosponsored by the AIAA, ASME, SAE, and ASEE Indianapolis, Indiana, June 27-29, 1994

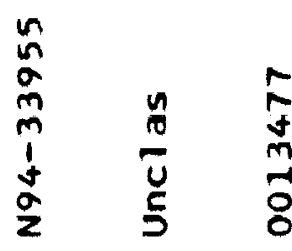

$n$
$n$
0
0
0
0

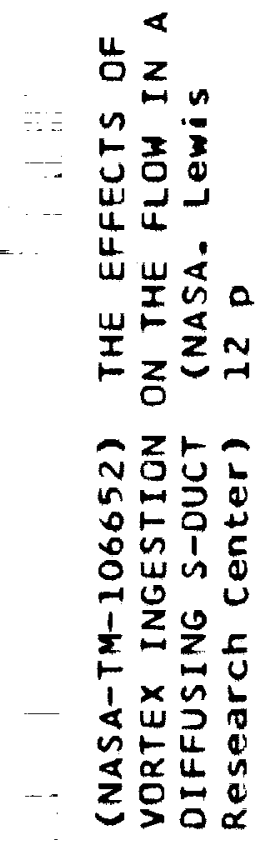




\title{
THE EFFECTS OF VORTEX INGESTION ON THE FLOW IN A DIFFUSING S-DUCT
}

\author{
B. J. Wendt" and B. A. Reichert ${ }^{\dagger}$ \\ NASA Lewis Research Center, Cleveland, Ohio
}

\begin{abstract}
An experimental study of the effects of an ingested vortex on the flowfield of a diffusing S-duct is reported. The vortex is generated through the use of a stationary pinwheel device mounted upstream of the diffusing Sduct. Three test conditions vary the location of the vortex in the duct inlet crossplane. For each condition of ingested vortex, a baseline S-duct and an S-duct with an array of vortex generators is examined. The data taken consist of duct inlet and exit crossplane surveys of velocity and total pressure. Duct surface flow visualization and static pressure are also recorded. The data acquired in these tests are compared to identical S-duct data taken in the absence of the ingested vortex.

The ingested vortex is observed to have a strong influence on the flowfield inside (and exiting) the S-duct, but only when the vortex impinges at the inlet crossplane location coincident with the crossplane location of downstream flow separation within the duct. When the ingested vortex impinges at this location it reduces the extent of flowfield separation inside the baseline duct and promotes stronger crossflow in the exit plane of both the baseline duct and the duct with installed vortex generators. This enhanced crossflow also strengthens the vortices shed from the vortex generators. The other impingement locations of the ingested vortex are found to produce little effect on the flowfield of the duct, with or without vortex generators.
\end{abstract}

\section{Introduction}

An initially uniform airstream enters an aircraft inlet and is routed to the engine face. Inlet performance, in terms of total pressure recovery and total pressure distortion, is degraded by flow phenomena originating within the duct itself. The subject of internally generated distortion and recovery losses is generally well documented in the literature for a variety of inlet duct types. Relatively little information exists on flow problems occurring within inlet ducts due to non-uniform upstream conditions. One type of upstream non-uniformity is a trailing

\footnotetext{
-Research Engineer, National Research Council Associate. Member AIAA.

${ }^{\dagger}$ Research Engineer, Inlet, Duct, and Nozzle Flow Physics Branch. Member, AIAA.

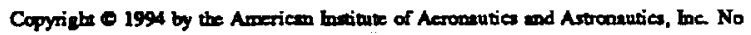

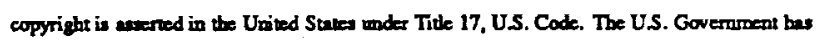
arylty-fice licenes to excrcive all righe under the copyright elaimed berein for Governmentel

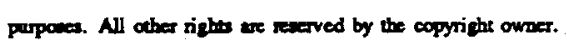

vortex that has been ingested into the inlet. Such a vortex may be shed, for example, from a forward component of the airframe during a high angle-of-attack maneuver. In a recent computational study by Anderson ${ }^{1}$ the effects of an ingested vortex on the flow field within a F/A-18 inlet duct were examined. Anderson observed that the vortex trajectory within the duct behaves as though the flow field were inviscid, and that for some values of strength and location the ingested vortex negates the beneficial flow control effects provided by vortex generators installed on the duct surface.

The objective of the present study is to experimentally explore the effects of an ingested vortex on the flow field within a diffusing S-duct Two different configurations of the diffusing S-duct are considered, the baseline S-duct, and the same duct with an array of surface mounted vortex generators.

The flow features of the baseline configuration were explored in studies by Vakili $e t a l^{2}$ and Wellborn $e t$ $a l .^{3} \quad$ The primary features of interest originate with strong cross-stream pressure gradients developed by the duct centerline curvature. Imposed on the duct boundary layer, these pressure gradients give rise to secondary flows which converge near the duct surface at the angular position of flow field symmetry. This convergence, combined with an increasing cross-sectional area, results in a three-dimensional boundary layer separation. The resulting secondary flows develop into a pair of counterrotating axial vortices at the duct exit. These vortices are responsible for a good deal of total pressure distortion at the engine face. Additionally, flow separation contributes to the flow blockage that reduces the total pressure recovery of the duct.

As shown in recent experimental work by Reichert and Wendt ${ }^{4}$ the duct distortion may be significantly reduced and total pressure recovery increased if vortex generators are used to "counter" or redirect the secondary flow converging near the duct surface. Boundary layer flow separation is eliminated in the duct and the counterrotating vortices found at the duct exit are weakened, thereby reducing their deleterious effects on the flow field. Eight different vortex generator arrays were tested in Ref. 4. The vortex generator array that produced the best total pressure recovery and least distortion was used in this study.

The results for three different conditions of ingested vortex are reported here. The ingested vortex conditions vary by the location of the vortex at the upstream boundary of the duct test section. For each condition of ingested vortex, a baseline S-duct and an S-duct with an array of vortex generators is tested. The data presented for each 


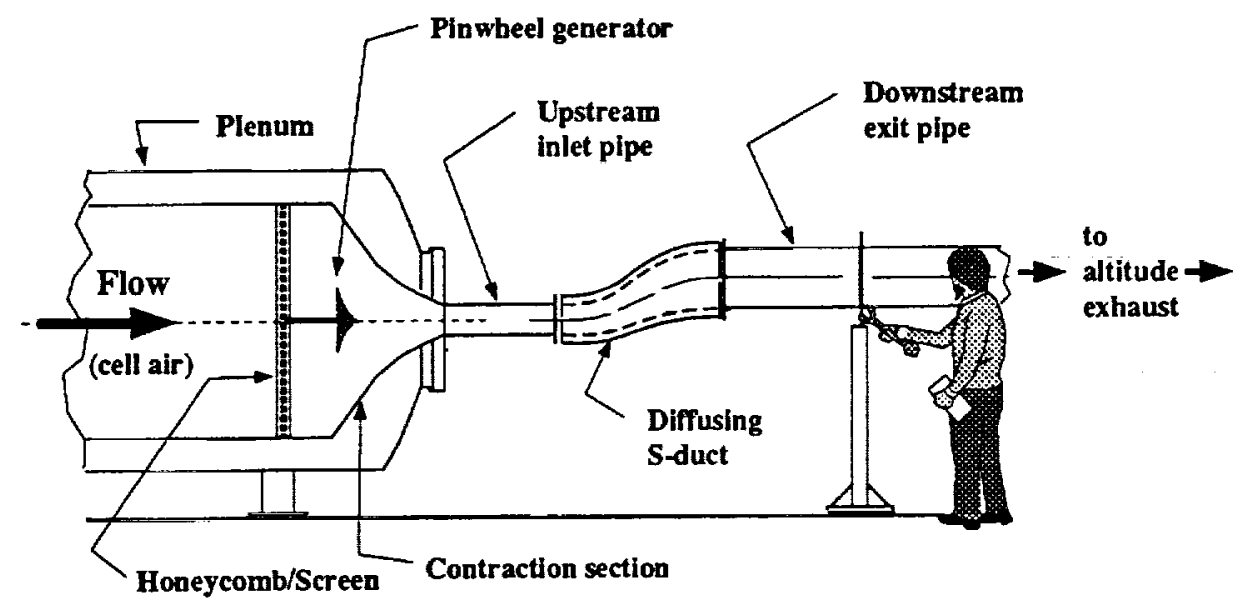

Fig. 1 The test section region of the IFMF showing the approximate mounting location of the pinwheel (ingested) vortex generator.

condition includes total pressure and transverse velocity fields acquired at full duct inlet and exit crossplanes, as well as duct surface flow visualization and static pressure data. These data are compared with the data acquired in the previous baseline and vortex generator tests, Refs. 3 and 4.

In the first condition, the center of the ingested vortex coincides with the centerline of the inlet crossplane. Measurements of flow angularity, transverse velocity, and total pressure at the upstream crossplane aid in characterizing the ingested vortex initial strength and structure.

In the second condition, the center of the ingested vortex impinges near the wall of the inlet crossplane, at the circumferential location where strong secondary flows converge downstream to originate boundary layer separation.

In the third condition, the center of the ingested vortex impinges on the inlet crossplane near the duct wall opposite that of the second condition.

\section{Experimental Facilities and Procedures}

\section{Facility Flow Conditions}

Experimental measurements of the duct flow field were made at NASA Lewis Research Center using the Intemal Fluid Mechanics Facility (IFMF). This facility was designed to support the research of a variety of internal flow configurations and is described in detail by Porro et al. ${ }^{5}$ The facility is schematically illustrated in Fig. 1. As shown in Fig. 1, smooth circular pipes of appropriate diameter were attached upstream and downstream of the S-duct to produce (in the absence of the "pinwheel" described below) a uniform incoming flow and a smooth, continuous condition for flow exiting the duct. The lengths of the upstream and downstream pipes were each $3.75 D_{1}$ where $D_{1}$ is the diameter of the S-duct at the inlet crossplane. The duct inlet Mach number was $M=0.6$ for all experimental test conditions and measurements. The inlet boundary layer thickness was approximately $4 \%$ of the duct inlet diameter and the Reynolds number, based on inlet diameter, was $R e_{D_{1}}=$ $2.6 \times 10^{6}$.

The ingested vortex was generated with a nonrotating eight bladed pinwheel. The triangular blades of the pinwheel were cut from an eighth-inch sheet of aluminum rolled to an inside diameter of 24 inches. The eight blades were welded to a single aluminum "hub" or ring to form the pinwheel pattern. The pinwheel was mounted on a sting anchored to a honeycomb-screen combination located at the downstream end of the facility plenum section. The sting and pinwheel extended downstream about 14 inches into the contraction section of the tunnel as indicated in Fig. 1. The pinwheel and sting are illustrated in Fig. 2. The position of the ingested vortex within the duct could be varied by mounting the sting and pinwheel at different cross-stream locations on the honeycomb-screen combination. When looking upstream into the inlet pipe, the ingested vortex is seen to be a counter-clockwise vortex.

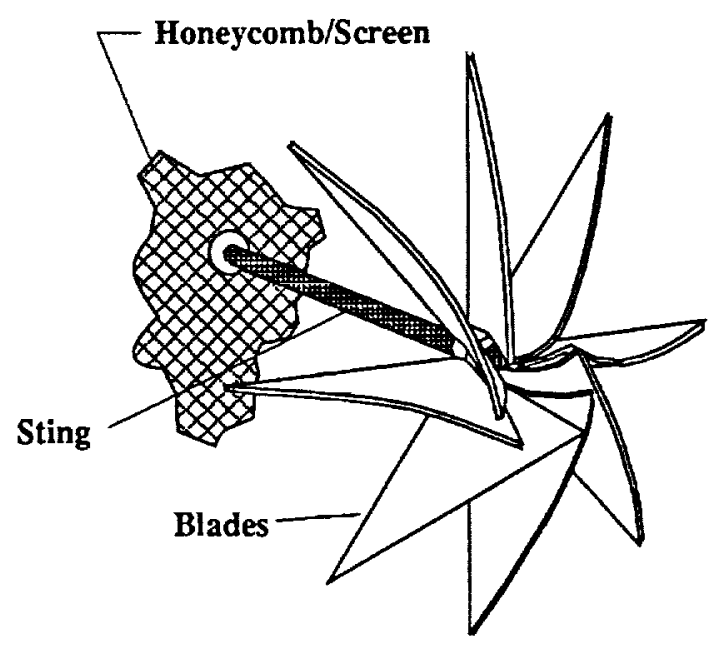

Fig. 2 The ingested vortex generator pinwheel. 
Baseline Diffusing S-Duct

The geometry of the baseline diffusing S-duct examined in this study is shown in Fig. 3. This duct is geometrically similar to the duct tested in Ref. 2 and is identical to the duct studied in Refs. 3 and 4. The duct centerline is defined by two circular arcs with an identical radius of curvature, $R=102.1 \mathrm{cms}$, and subtended angle of $30^{\circ}$. Both arcs lie within the $x z$ plane as shown in Fig. 3. The cross-sectional shape of the duct perpendicular to the centerline is circular. When discussing locations within the duct, axial location will refer to distance to cross-stream planes measured along the duct centerline and normalized by the duct inlet diameter, $s / D_{1}$. Position within cross-stream planes is specified by the polar angle $\phi$, measured from the vertical in a positive clockwise direction as shown in Fig. 3, and the radial distance from the centerline, $r$. The diameter of the cross-section varies with the axial location as follows:

$$
\begin{aligned}
\frac{D}{D_{1}} & =1+3\left(\frac{D_{2}}{D_{1}}-1\right)\left(\frac{s / D_{1}}{5.23}\right)^{2} \\
& -2\left(\frac{D_{2}}{D_{1}}-1\right)\left(\frac{s / D_{1}}{5.23}\right)^{3}
\end{aligned}
$$

In Eq. (1) and Fig. 3, $D_{1}=20.4 \mathrm{cms}$ is the diameter at the duct inlet and $D_{2}=25.1$ is the diameter at the duct exit. This provides an exit to inlet area ratio of $A_{2} / A_{1}=$ 1.52. The offset of the duct resulting from the centerline curvature is $1.34 D_{1}$, and the length of the duct measured along the centerline is $5.23 D_{1}$. A plane which runs parallel to the duct centerline and intersects the angular positions of $\phi=0^{\circ}$ and $180^{\circ}$ is the aforementioned plane of flow field symmetry for the baseline test case.

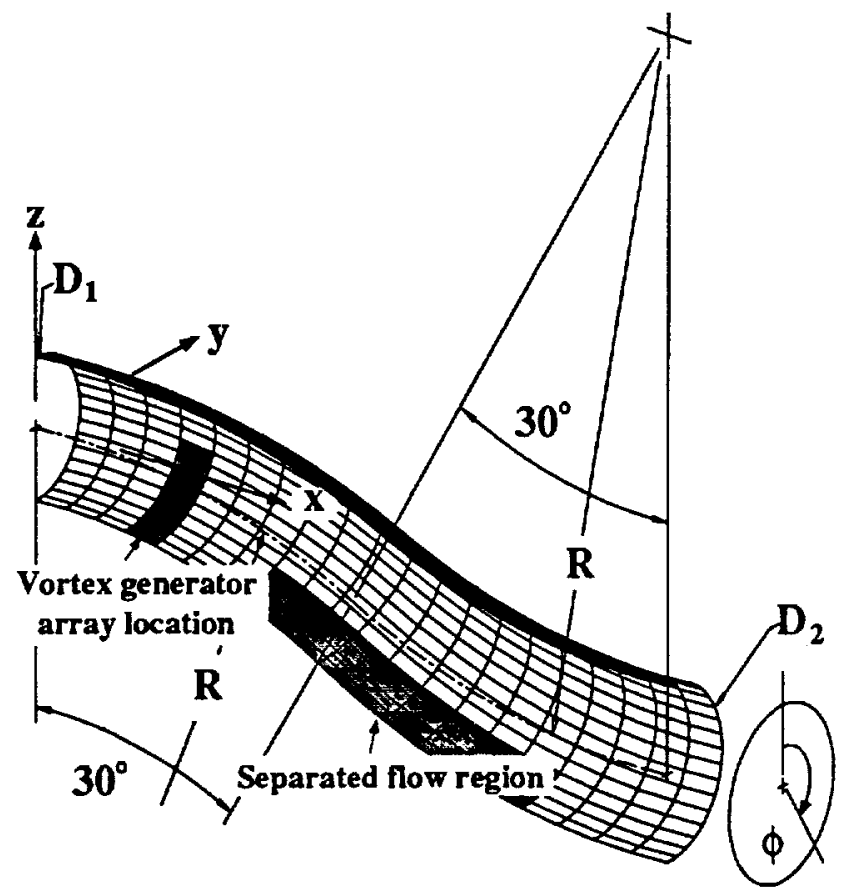

Fig. 3 The geometry of the diffusing S-duct.

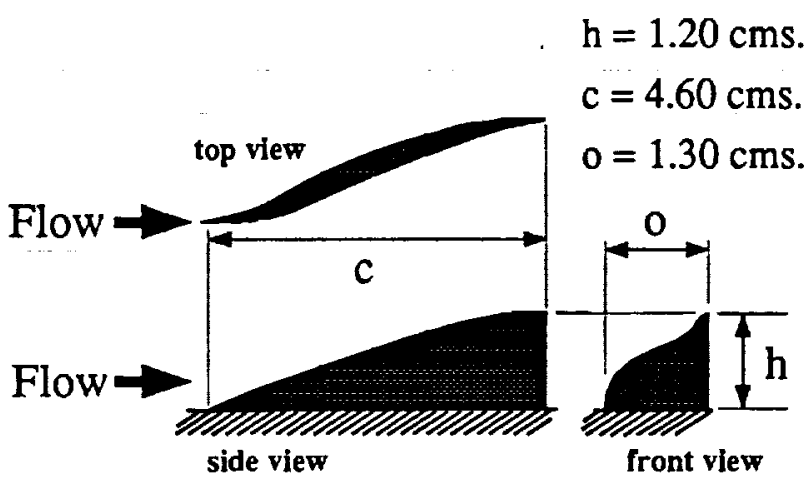

Fig. 4 The geometry of the tapered-fin vortex generator used inside the S-duct.

The approximate streamwise location of boundary layer separation in the baseline duct is indicated in Fig. 3. Also shown is the approximate mounting position used for vortex generators in the duct.

\section{Diffusing S-Duct with Vortex Generators}

The vortex generators used are illustrated in Fig. 4. These devices are similar to the "tapered fin" first examined in the report of Schubauer and Spangenberg. ${ }^{6}$ Each vortex generator will produce a single trailing axial vortex when its leading edge is aligned with the flow as indicated in Fig. 4. The height of the vortex generators tested were on the order of the flow field boundary layer thickness.

The basis for flow control using arrays of these devices can be understood from flow visualization data obtained in the baseline diffusing S-duct. Figure 5 illustrates surface flow visualization results, shown as dashed lines, obtained by using oil dots. Upstream of the axial loca-

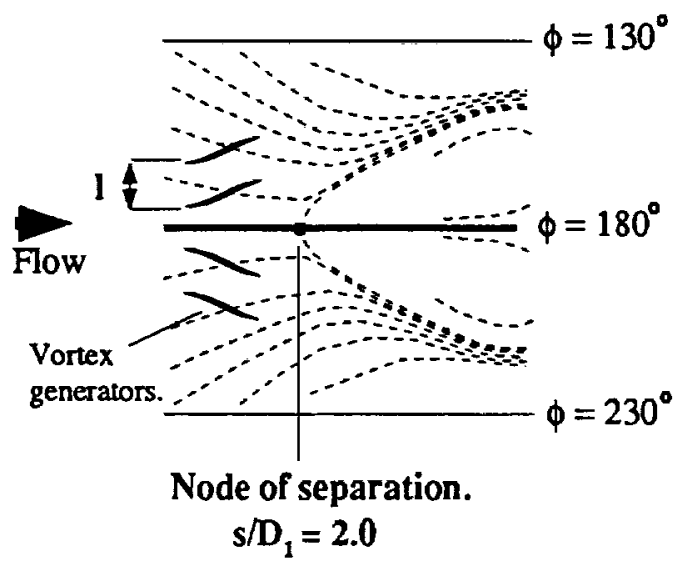

Fig. 5 Vortex generators are positioned to counter the converging flow near the node of separation. The parameter "P" is the cross-stream spacing between generators.

tion of flow separation $\left(s / D_{1}=2.0\right)$ in the angular range $80^{\circ} \leq \phi \leq 280^{\circ}$ the flow is converging strongly toward the line of flow field symmetry, $\phi=180^{\circ}$. Continuity 
forces these converging flows away from the duct surface near $\phi=180^{\circ}$. This motion initiates the naturally occurring pair of counter-rotating vortices observed at the duct exit for flow without installed vortex generators. Also, the converging flow of low momentum fluid thickens the boundary layer near $\phi=180^{\circ}$ and reduces its ability to withstand streamwise adverse pressure gradients, contributing to flow separation. The vortex generators are mounted in arrays to counter this converging flow, as shown in Fig. 5. As discussed in Ref. 4, the array of $N=4$ vortex generators with a cross-stream spacing of $l / D_{1}=0.16$ (as shown in Fig. 5) is an optimum array in the sense that it produces minimal flow distortion and maximum total pressure recovery at the exit plane of the S-duct. This array of vortex generators eliminated boundary layer separation within the duct.

\section{Measurement Techniques}

The primary set of measurements consist of inlet and exit crossplane surveys of the mean three-dimensional velocity field and total pressure. The inlet survey crossplane was located a distance $0.5 D_{1}$ upstream of the S-duct inlet. The survey grid at this location consisted of 9 radial traverses across the diameter of the pipe. Each radial traverse consisted of 32 equally spaced measurements. Resolution along the radial axis was $\Delta r / D_{1}=0.031$ and $\Delta \phi=20^{\circ}$ circumferentially. The data were acquired using a single five-hole probe. The exit measurement plane was located at $s / D_{1}=5.73$ and is shown in Fig. 6 . The

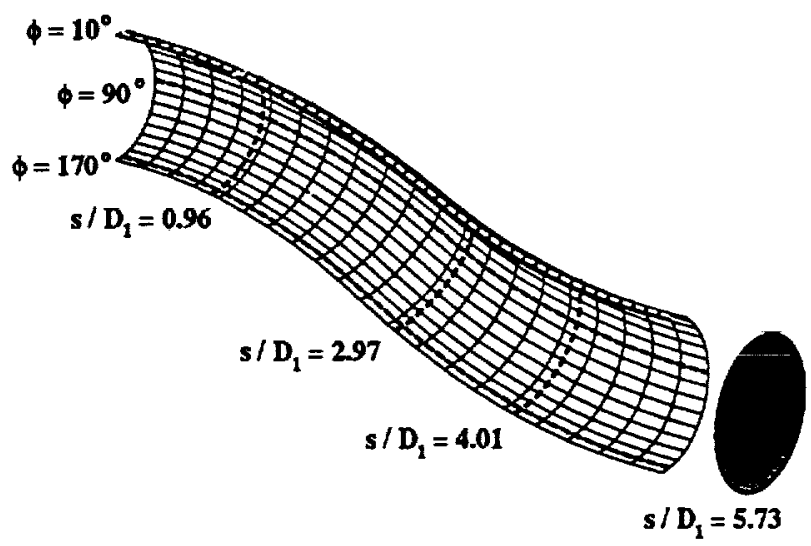

Fig. 6 Location of axial and circumferential static pressure taps and the exit measurement plane.

velocity and total pressure data were acquired here with a rake probe consisting of ten equally spaced and independently calibrated five-hole probe tips. The rake probe was traversed radially and circumferentially to acquire data at 720 uniformly spaced grid points in the $(r, \phi)$ exit plane survey grid. Grid resolution on the radial axis was $\Delta r / D_{2}=0.025$ and $\Delta \phi=10^{\circ}$ circumferentially. More information on the geometry, construction, and calibration of the five-hole probe rake used in this study can be found in the report of Wendt and Reichert. ${ }^{7}$
In addition to the velocity field survey, visualization of the near-surface duct flow was conducted using a fluorescent oil dot technique. The flow pattern revealed by the oil dots was photographed under ultraviolet illumination. These pattems were then transferred (by contact) to absorbent paper so that two-dimensional tracings of the flow pattern could be rendered. Surface static pressures inside the S-duct were recorded by a grid of 220 taps located on axial lines at angles $\phi=10^{\circ}, 90^{\circ}$, and $170^{\circ}$, as well as circumferential lines at $s / D_{1}=0.96,2.97$, and 4.01. The locations of the static pressure taps are indicated by the dashed lines in Fig. 6 .

\section{Results and Discussion}

Static and total pressure data are plotted in terms of the corresponding non-dimensional pressure coefficients defined as follows:

$$
\begin{aligned}
C_{p} & =\frac{p-p_{\text {ref }}}{p_{o, \text { ref }}-p_{\text {ref }}}, \\
C_{p o} & =\frac{p_{0}-p_{\text {ref }}}{p_{o, \text { ref }}-p_{\text {ref }}},
\end{aligned}
$$

where $p_{0}$ and $p$ represent the local values of total and static pressure. Since the air being drawn through the IFMF test facility originates in the test cell, the reference value of total pressure, $p_{o, r e f}$, is atmospheric. The reference value of static pressure, $p_{r e f}$, is the average reading of the surface static pressure recorded near the inlet pipe survey plane, where 18 static pressure taps (spaced 20 degrees apart, around the pipe circumference) are recorded simultaneously.

Table 1 summarizes the test cases covered for this study. Two different S-duct configurations, a baseline duct and a duct with vortex generators, are charted against four upstream boundary conditions. Test cases 1 and 2 are without the ingested vortex and represent the reference cases. Test cases 3 through 8 are the ingested vortex cases as defined in Table 1.

\section{Upstream Results}

Figure 7 illustrates the upstream results for each condition of ingested vortex. The velocity scale is provided by the reference vector at the bottom of Fig. 7. This vector represents one-tenth the crossplane averaged total velocity magnitude. Figure $7 \mathrm{a}$ is a contour plot of total pressure at the inlet crossplane in the absence of the ingested vortex. The inset shows the uniform boundary layer present here.

Figure $7 \mathrm{~b}$ illustrates the transverse velocity and total pressure data for the impingement of the vortex on the duct centerline. A strong, coherent vortical structure is evident from the transverse velocity data, which has been interpolated from the radial survey grid to the Cartesian grid shown. Flow angles range between $\pm 10^{\circ}$. Vortex 


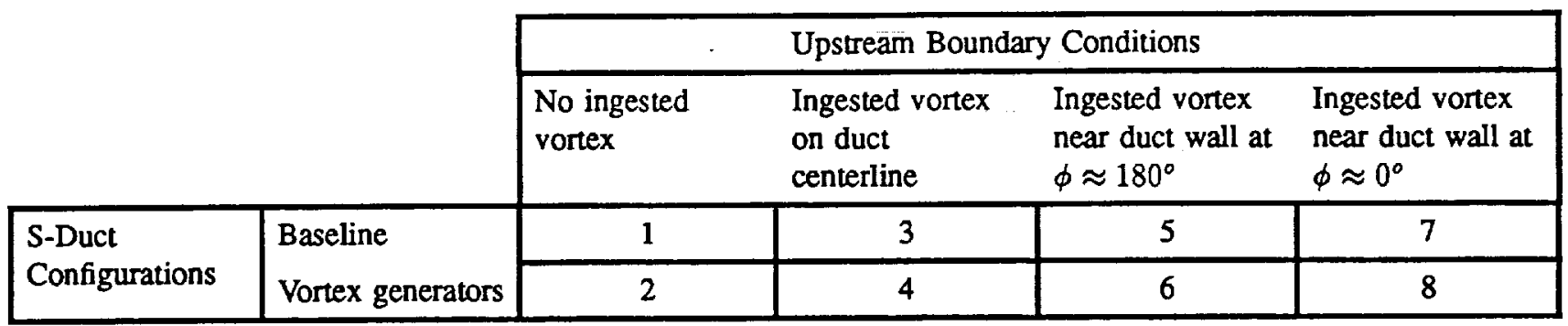

Tab̆le 1 A summary of test cases.

flow effects are confined to a sector roughly four inches in diameter and centered on $r=0$. The ingested vortex has no apparent effect on the total pressure field in Fig. $7 \mathrm{~b}$.

Figures $7 c$ and $7 d$ illustrate the off-centerline impingement of the ingested vortex. Figure $7 c$ presents the transverse velocity and total pressure results for test

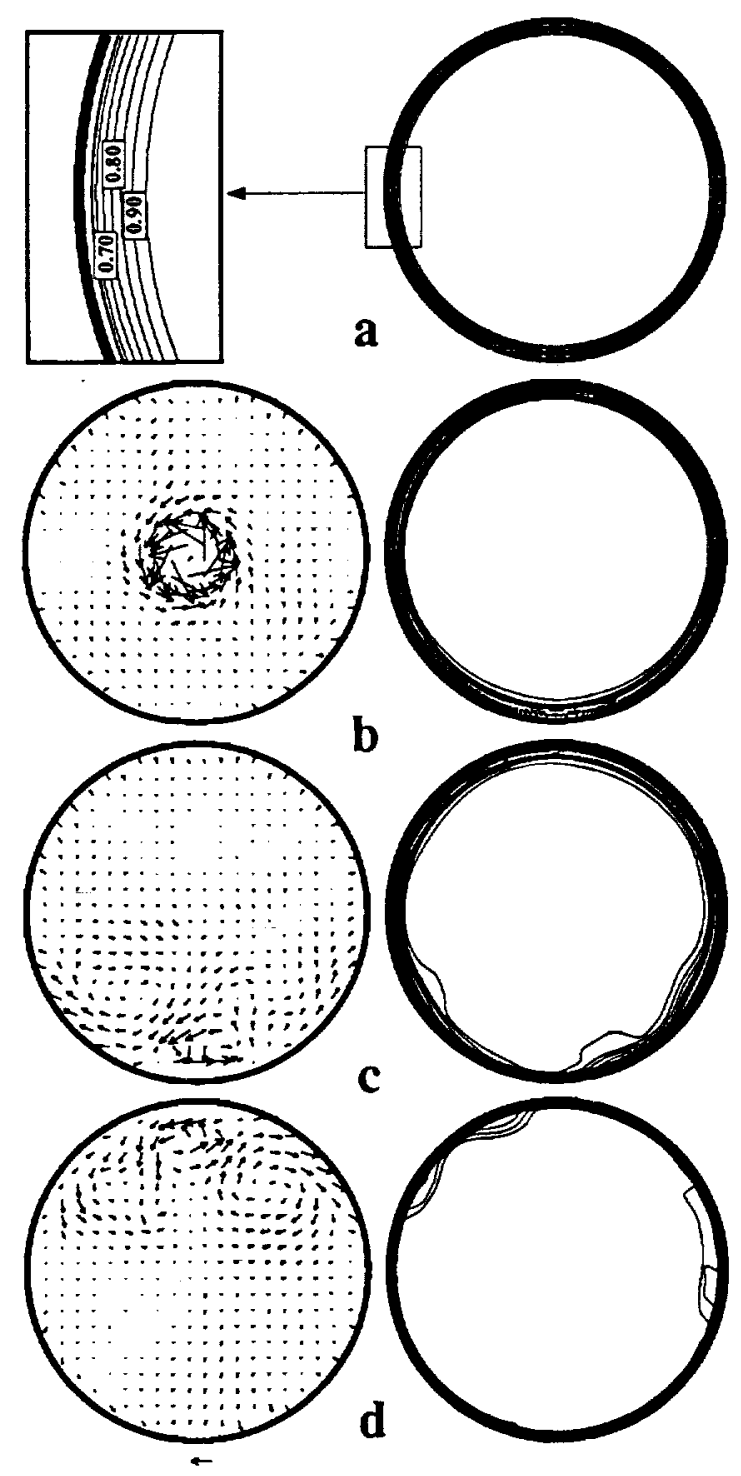

Fig. 7 Transverse velocity and total pressure results at the duct inlet plane (looking upstream). cases 5 and 6, Fig. $7 \mathrm{~d}$ for test cases 7 and 8 . The velocity data show a grouping of 3 distorted vortices for both of these impingement conditions. The center vortex (a counter-clockwise vortex, closest to the wall) is the strongest of the three. This vortex is the pinwheelgenerated vortex. The additional vortices in the flowfield are most likely the result of an interaction between the pinwheel-generated vortex and the upstream contraction surface boundary layer. Corresponding distortions in the boundary layer profiles are apparent in the total pressure profiles of Figs. $7 \mathrm{c}$-d.

\section{S-Duct Results}

Figure 8 illustrates the surface flow visualization results obtained inside the diffusing S-duct, near the region of flow separation. These two-dimensional patterns were obtained by pressing absorbent paper over oil flow patterns on the duct surface. In each figure the flow is from left to right.

Figures 8a-b represent the reference test cases 1 and 2 (see Table 1). The vortical region of flow separation is clearly evident in Fig. 8a. In the absence of the ingested vortex the baseline flowfield possesses mirror image symmetry through the line $\phi=180^{\circ}$, as discussed earlier. This is also true for the diffusing S-duct with a symmetric array of vortex generators installed as depicted in Fig. 5. Note the absence of separated flow in Fig. $8 \mathrm{~b}$.

Figures $8 \mathrm{c}, 8 \mathrm{e}$, and $8 \mathrm{~g}$ are the surface flow visualization results for the baseline $S$-duct with ingested vortex (test cases 3,5, and 7, respectively). The effect of the ingested vortex is seen to be strongest in test case 5. Flow stagnation on the surface is still evident over the axial range $2<s / D_{1}<4$ indicating flow separation, but the structure of this region is now different from reference test case 1. The mirror image symmetry of the vortical separation is destroyed, and a smaller, more concentrated, region of vortical flow appears on the surface, centered on the line $\phi=180^{\circ}$. Downstream, in the angular range $180^{\circ}<\phi<270^{\circ}$, the surface streaklines converge and trail off towards higher values of $\phi$ creating an asymmetric pattem in contrast to Fig. 8a. Crossflow created by the near-wall proximity of the ingested vortex is the probable cause of this effect. The flow visualization results for test cases 3 and 7 illustrate that these conditions of ingested vortex have little influence on the surface flow features of the duct in the region of flow separation. 


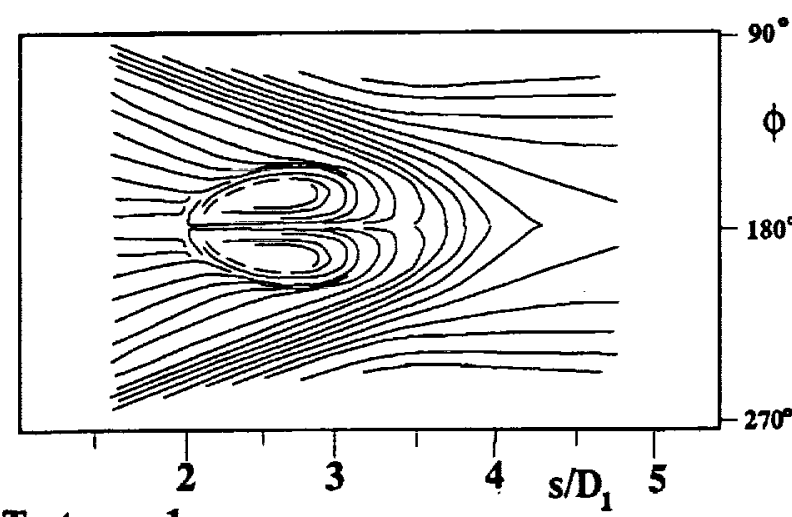

a) Test case 1 .

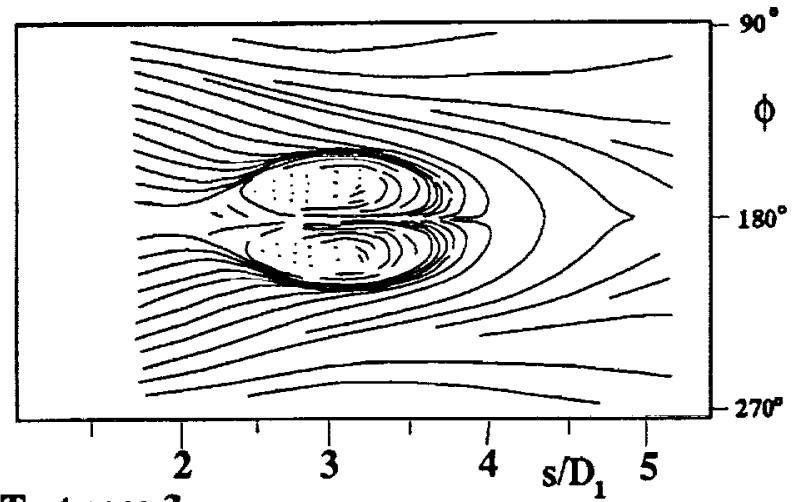

c) Test case 3.

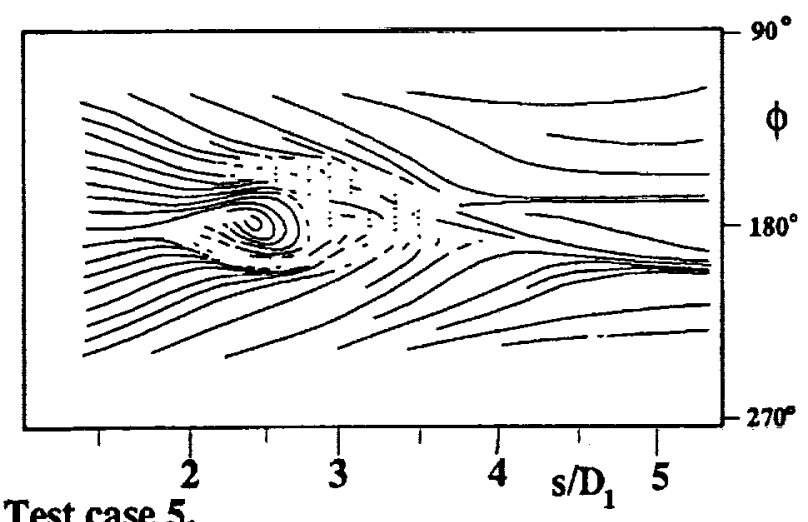

e) Test case 5.

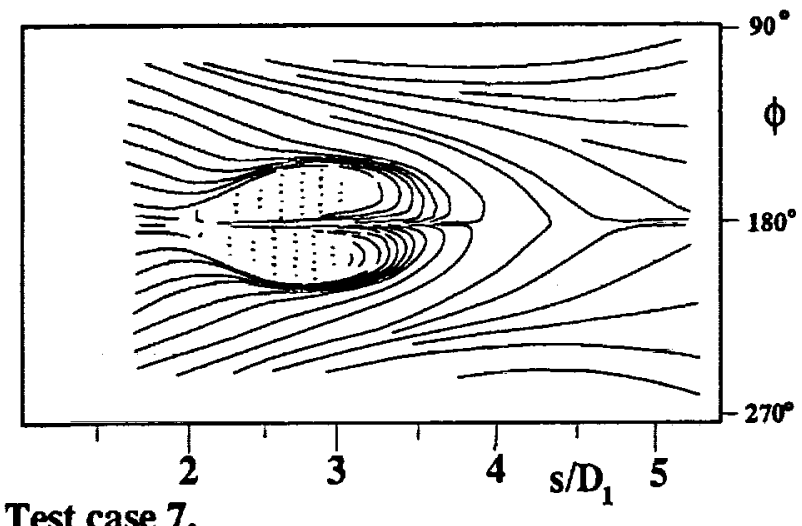

g) Test case 7.

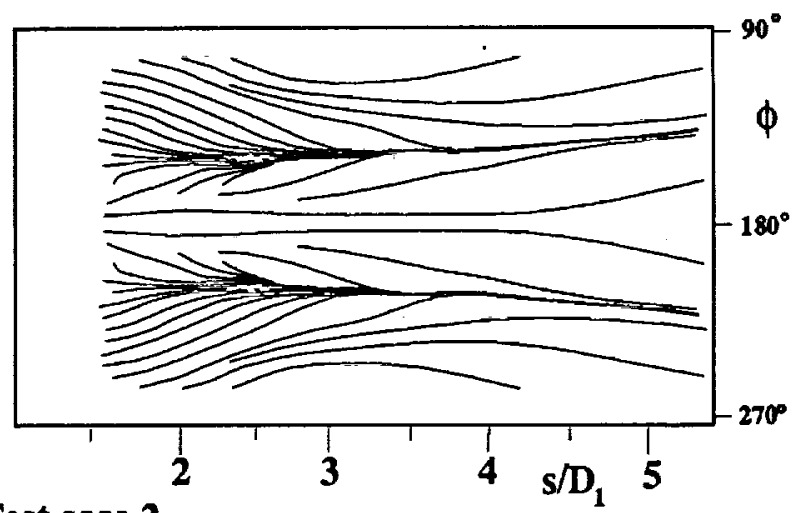

b) Test case 2 .

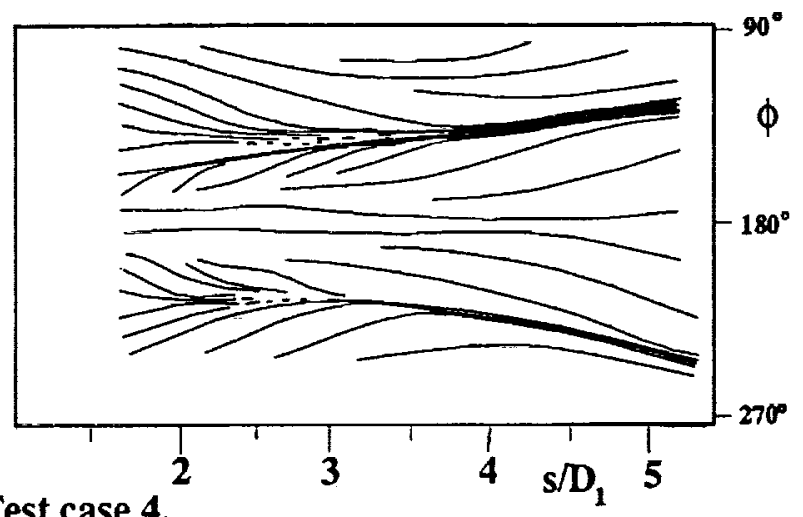

d) Test case 4 .

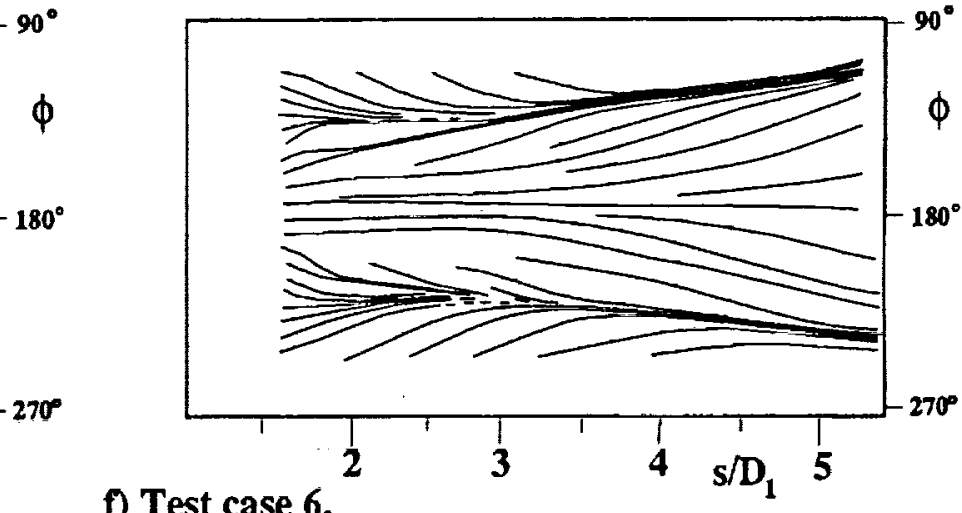

f) Test case 6.

h) Test case 8.

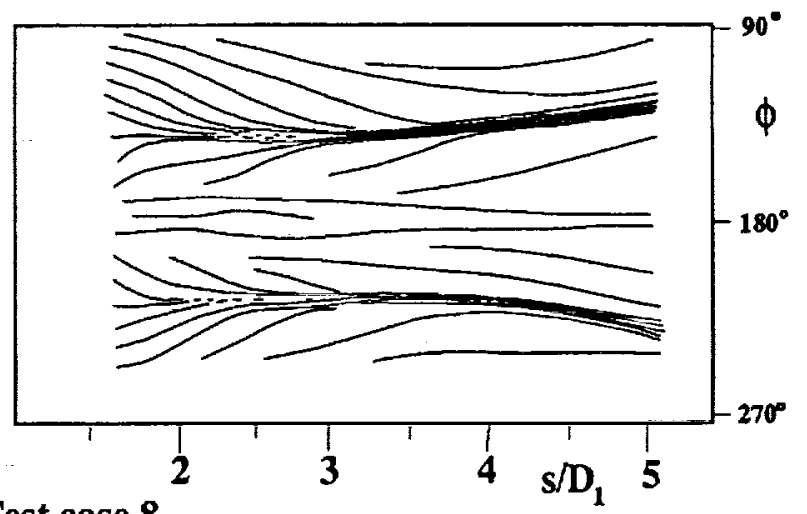

Fig. 8 Surface flow visualization results obtained in the diffusing S-duct.

Figures 8d, 8f, and $8 \mathrm{~h}$ are the surface flow results for the S-duct with an array of vortex generators (test cases 4,6 , and 8, respectively). Qualitatively, these present the same surface flow features seen in Fig. $8 \mathrm{~b}$. The divergence of the two concentrated patterns of converging streaklines (from $\phi=180^{\circ}$ ) results when the crossflow 
created by the vortex generator array counters the naturally occurring crossflow as discussed in the Introduction. The divergence of the two streakline patterns in Fig. $8 \mathrm{f}$ is somewhat greater than that observed in the other vortex generator test cases. We also have evidence, from Fig. 8e, that this condition of ingested vortex is creating a crossflow pattem sweeping down from $\phi=180^{\circ}$ towards higher values of $\phi$. Thus the ingested vortex is observed to enhance the crossflow created by that portion of the vortex generator array installed over $\phi>180^{\circ}$.

Figure 9 illustrates the surface static pressure results obtained in the S-duct for all test cases.

The ingested vortex test cases are plotted with filled symbols and are compared against the reference test cases, plotted using open symbols. Figures $9 \mathrm{a}-\mathrm{f}$ are the results for the baseline duct with ingested vortex, and these results are compared against test case 1. Figures $9 \mathrm{~g}-\mathrm{l}$ are the results for the S-duct with vortex generators, and these results are compared against test case 2 . In the plots of axial static pressure variation (Figs. 9a-c and $9 \mathrm{~g}-\mathrm{i})$ the vertical dashed lines denote the axial locations of the 3 rows of circumferential static taps. In the corresponding plots of circumferential static pressure variation (Figs. 9d-f and 9j-l) the vertical dashed lines represent the angular position of the 3 rows of axial taps.

Consider, first, the axial pressure variation for test case 1, plotted using the open symbols in Figs. 9a-c. The constant values of static pressure found in the range $2<s / D_{1}<3$ at $\phi=90^{\circ}$ and $170^{\circ}$ are a result of flow separation at these locations. The effect of separated flow is also evident in the circumferential static pressure results at $s / D_{1}=2.97$ and 4.01. At these axial positions, in the absence of flow separation, static pressure should rise monotonically to peak values at $\phi=180^{\circ}$. We observe, however, a flat response for $120^{\circ}<\phi<180^{\circ}$.

The effect of the ingested vortex on the baseline duct is strongest in test case 5 (Figs. 9b and 9e). Flow separation, represented by the flat pressure response recorded in test case 1 , is observed to be alleviated (somewhat) in Figs. 9b and 9e. Again, as was observed in the flow visualization results, the probable cause is the crossflow created by the close proximity of the ingested vortex to the region of flow separation in test case 5. In Figs. 9a, 9c, 9d, and 9f the data overlap, also confirming what we observed in the flow visualization results, namely, that the ingested vortex is not affecting the surface flow features of the baseline duct for test conditions 3 and 7 .

Figures $9 \mathrm{~g}-1$ present corresponding results for the $\mathrm{S}$ duct with vortex generators. Reference test case 2 (open symbols in Figs. 9g-l) demonstrates the effectiveness of the vortex generators in alleviating the effects of flow separation on static pressure recovery. The data nearly overlap for test cases including the ingested vortex (test cases 4,6 , and 8) confirming results obtained from flow visualization.
Downstream Results

Figure 10 illustrates the exit plane transverse velocity and total pressure results for all test cases. Figure $10 \mathrm{a}$ is the baseline reference (test case 1), and Figs. 10b$\mathrm{d}$ are the baseline cases with ingested vortex. As with the upstream velocity data, the transverse velocity scale is provided by a reference vector above each plot. The interpretation of this vector is the same as before.

The transverse velocity field of Fig. 10a clearly shows the naturally occurring pair of counter-rotating upflow vortices. The term "upflow" refers to the convective action of the flow between the vortex cores. Low momentum fluid in the boundary layer region of the duct flowfield is convected up into the core flow by the vortices. The resulting distortion of the total pressure field is depicted in Fig. 10a. The flowfield possesses mirror image symmetry with respect to a line passing through the duct walls at the circumferential positions of $\phi=0^{\circ}$ and $180^{\circ}$.

The ingested vortex is clearly visible in the transverse velocity field of Fig. 10b. The effects of the ingested vortex on the total pressure field in test case 3 is minimal, however. In the transverse velocity field of Fig. 10c, no vortex structure identifiable as an ingested vortex is visible, but we clearly see the convective influence of this vortex. Note the shift in the position of the naturally occurring upflow vortices, to the right of center. This shift, due to the convective influence of the counter-clockwise ingested vortex, is also apparent in the total pressure contours. Note also that the low momentum "mound" of upflow fluid is reduced somewhat in size. The other impingement location of the ingested vortex, depicted in Fig. 10d, does not influence the exit plane results to the same extent exhibited in Fig. 10c. A small vortex structure, identified as the ingested vortex, is evident at the $100^{\prime}$ clock position near the wall in the transverse velocity field of Fig. 10d. The influence of the ingested vortex on the total pressure contours here is seen as a distortion in the boundary layer on the wall opposite to the upwelling of boundary layer fluid created by the naturally occurring vortices.

Figure 10e illustrates the transverse velocity and total pressure results at the exit crossplane of the Sduct with an array of vortex generators and no ingested vortex (test case 2). In the transverse velocity field we observe four vortices; the exterior vortices are the naturally occurring pair discussed earlier, and the interior vortices are created by the upstream array of vortex generators. Note the strong downflow now present on the line of flowfield symmetry. The effect on the total pressure contours is to split the mound of low momentum fluid, seen in Fig. 10a, into two smaller mounds displaced to either side of the symmetry line.

Figures 10f-h illustrate the effect of the ingested vortex on this flowfield. In the transverse velocity field of Fig. 10f we clearly see the presence of the centrally 

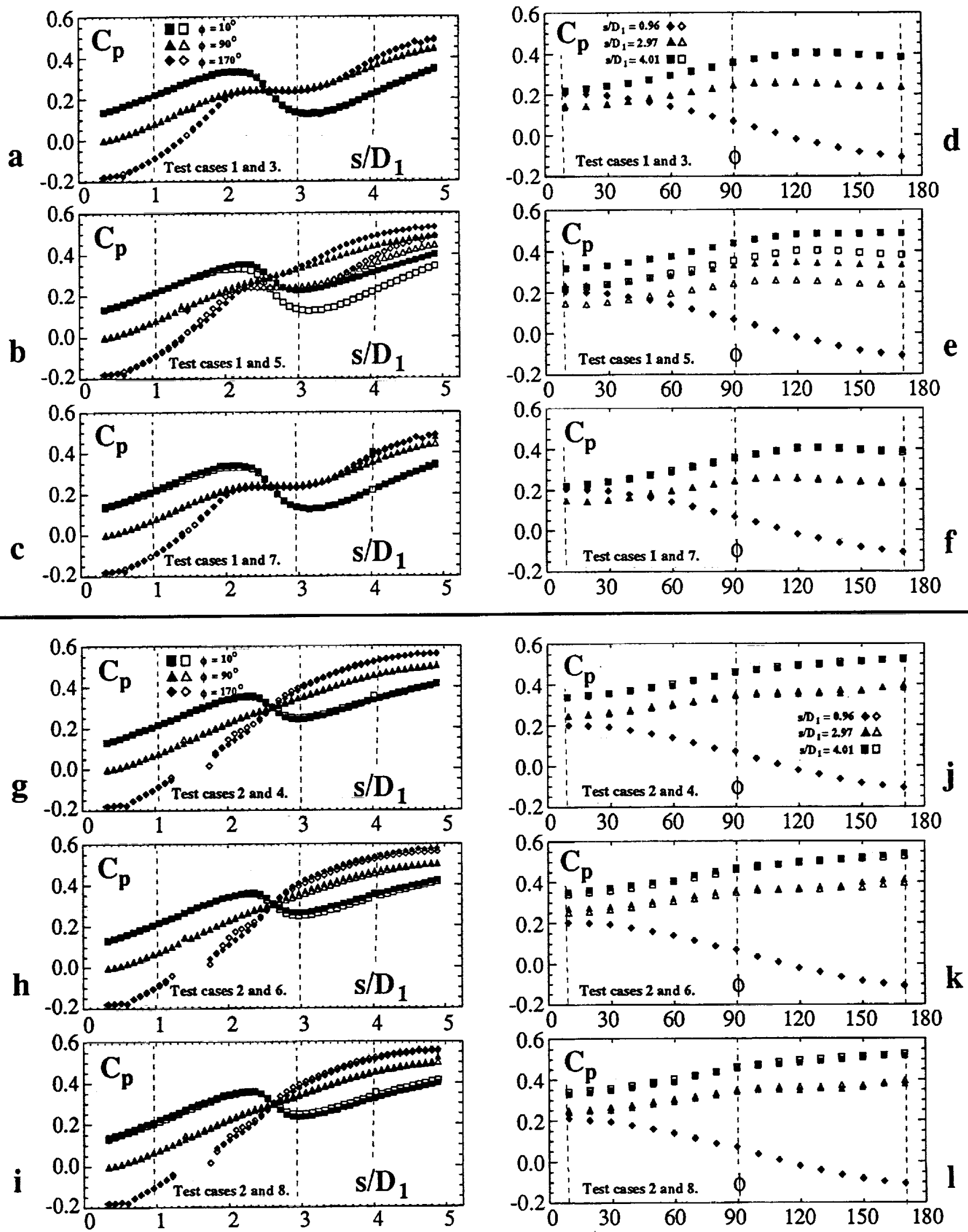

Fig. 9 Surface static pressure results obtained in the diffusing S-duct. 


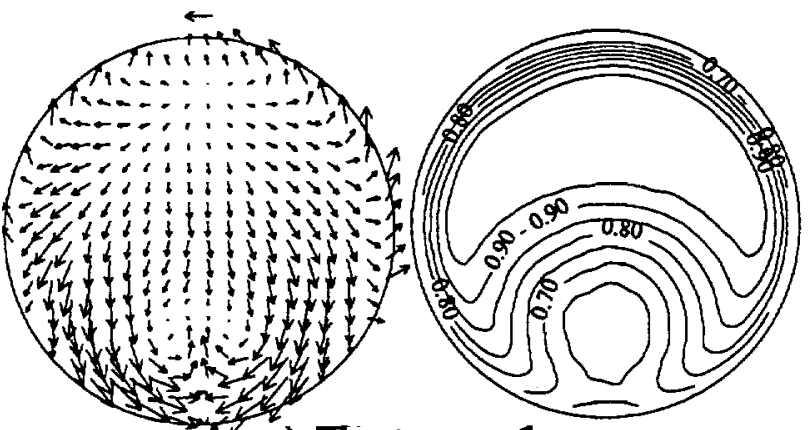

a) Test case 1 .

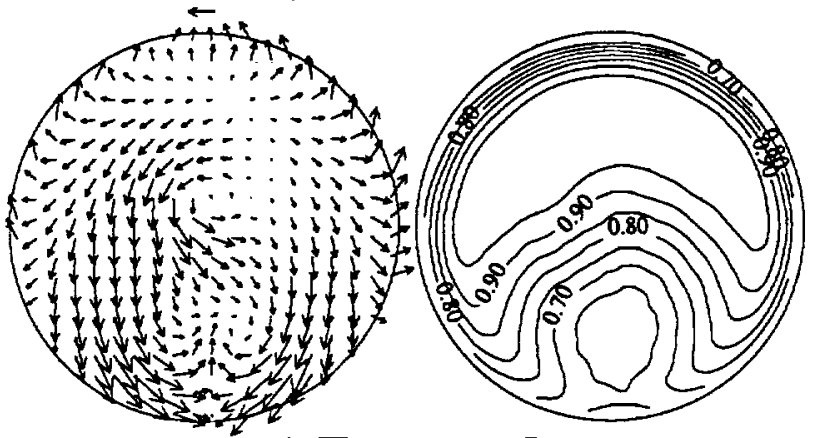

b) Test case 3 .

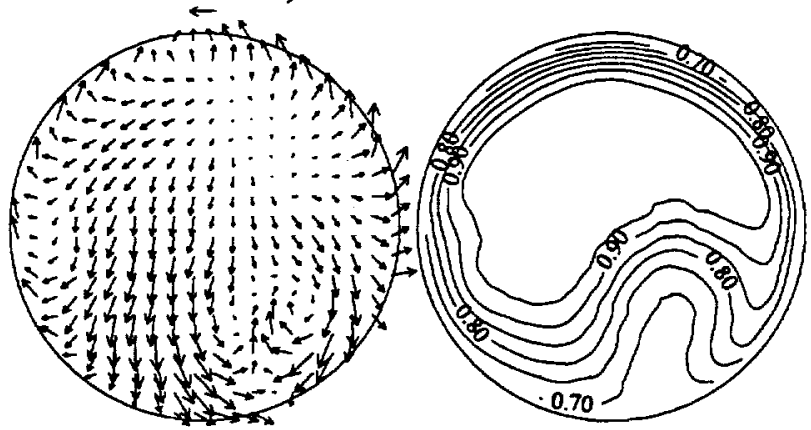

c) Test case 5 .

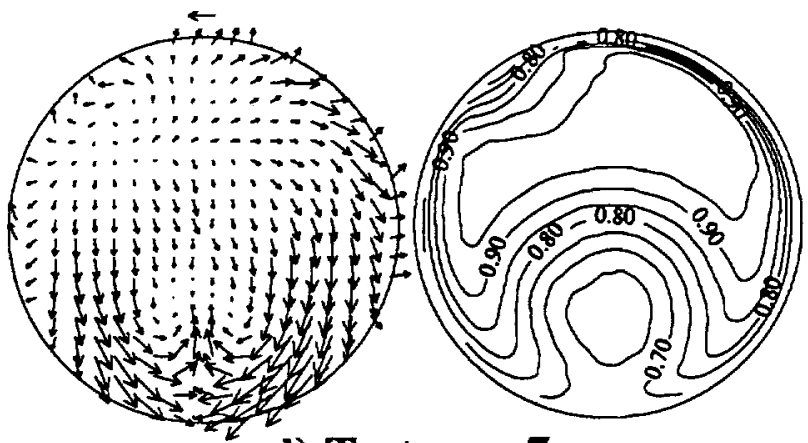

d) Test case 7.

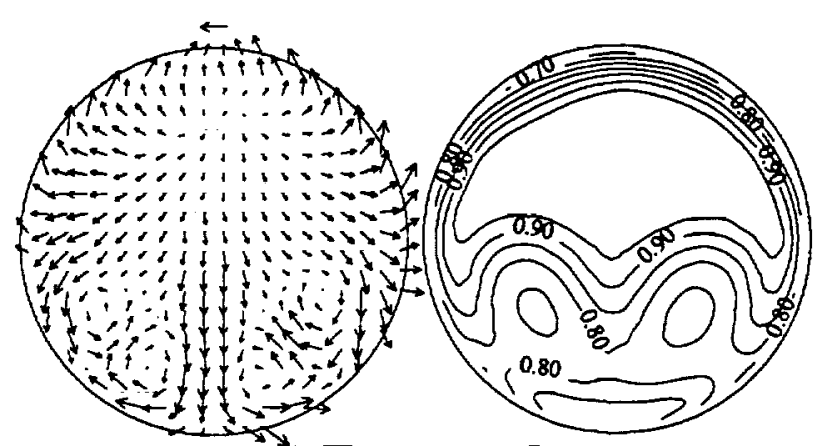

e) Test case 2.

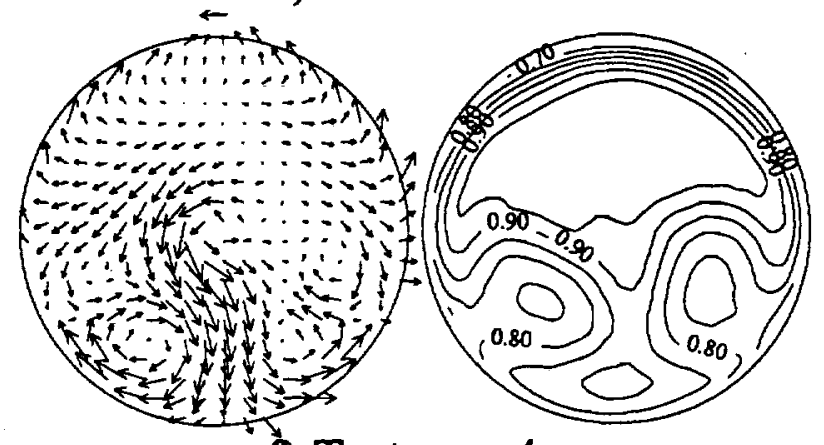

f) Test case 4.

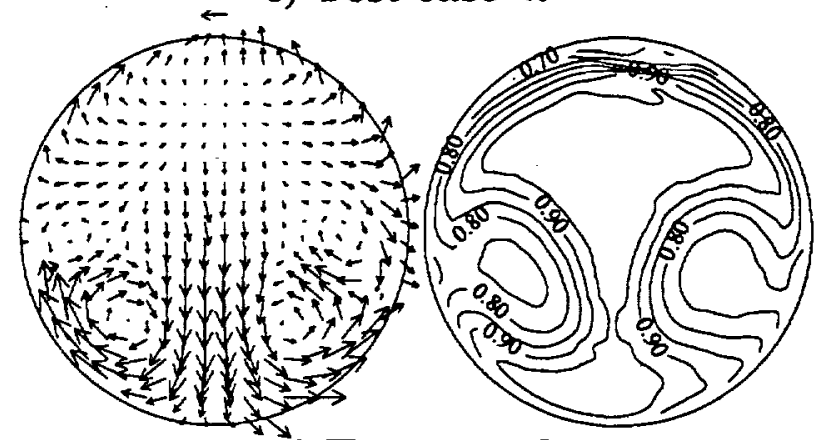

g) Test case 6.

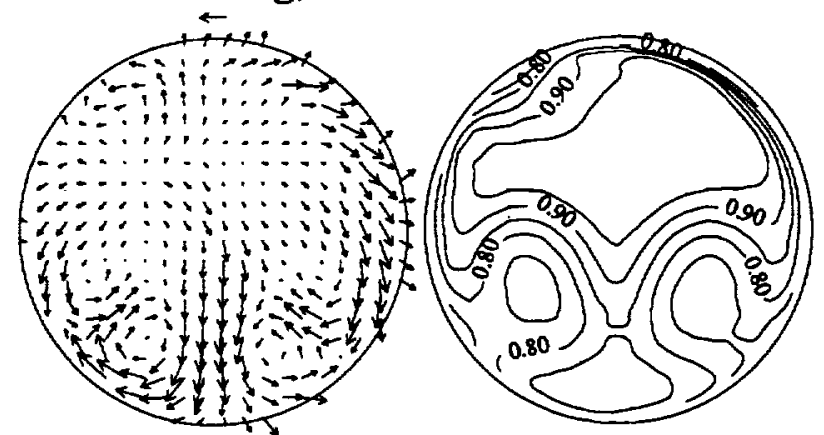

h) Test case 8.

Fig. 10 Transverse velocity and total pressure results at the duct exit plane.

located ingested vortex. The corresponding total pressure contours indicate that this vortex is having little effect on these results. Figure $10 \mathrm{~g}$ verifies what we deduced from flow visualization inside the duct : the ingested vortex is enhancing the effects of the vortex generator array on the flowfield. Note that the two interior vortices (due to the generators) and the region of downflow on the previous symmetry line are now much stronger than that observed in Fig. 10e. Figure 10h is similar to the pattern depicted in Fig. 10e, except for the small distortion in the boundary layer on the wall opposite the location of the vortices. This result is analogous to that occurring in the baseline duct for this condition of ingested vortex (Fig. 10d).

\section{Summary}

An experimental study of the effects of an ingested 
vortex on the flowfield of a diffusing S-duct is reported. The vortex is generated through the use of a stationary pinwheel device mounted upstream of the diffusing Sduct. Three test conditions vary the location of the vortex in the duct inlet crossplane. For each condition of ingested vortex, a baseline S-duct and an S-duct with an array of vortex generators is tested. The data taken consist of duct inlet and exit crossplane surveys of velocity and total pressure combined with duct surface flow visualization and static pressure. The data acquired in this test are compared to identical S-duct data taken in the absence of the ingested vortex.

In the first condition the ingested vortex impinges on the center of the duct inlet crossplane. Measurements of flow angle, transverse velocity, and total pressure at both inlet and exit crossplanes indicate a strong central vortex, with flow angles between $\pm 10^{\circ}$. When compared to identical results taken in the absence of the ingested vortex, little effect on the inlet and exit crossplane total pressure contours and S-duct surface flow behavior (as defined by surface static pressure and flow visualization) was evident.

In the second condition, the ingested vortex impinges near the duct wall on the inlet crossplane, in a region of the flowfield that develops separation downstream. This impingement location of the ingested vortex promotes stronger regions of transverse flow in the duct exit plane. Profiles of total pressure and surface flow features differ considerably from test cases without the ingested vortex. The strength of the vortices shed from the array of vortex generators is enhanced by the convective influence of the ingested vortex.

In the third condition, the ingested vortex impinges near the duct wall opposite that of the second condition. When compared to identical results taken in the absence of the ingested vortex, little effect on the inlet and exit crossplane total pressure contours and S-duct surface flow behavior (as defined by surface static pressure and flow visualization) was evident.

\section{Acknowledgments}

We are grateful for the help and insight provided by W. Darby, F. Forsyth, B. Gronski, W. Hingst, C. Wasserbauer, and K. Zaman.

\section{$\underline{\text { References }}$}

${ }^{1}$ Anderson, B. H., "The Aerodynamic Characteristics of Vortex Ingestion for the F/A-18 Inlet Duct," AIAA Paper 91-0130, Jan. 1991.

${ }^{2}$ Vakili, A. D., Wu, J. M., Liver, P., and Bhat, M. K., "Experimental Investigation of Secondary Flows in a Diffusing S-Duct," University of Tennessee Space Institute Tech. Rep. UTSI 86/14, 1984.

${ }^{3}$ Wellbom, S. R., Reichert, B. A., and Okiishi, T. H., "Aerodynamic Measurement of the Subsonic Flow Through a Diffusing S-Duct," AIAA Paper 92-3622, July 1992.

${ }^{4}$ Reichert, B. A. and Wendt, B. J., "Improving Diffusing S-Duct Performance by Secondary Flow Control," AIAA Paper 94-0365, Jan. 1994.

${ }^{5}$ Porro, A. R., Hingst, W. R., Wasserbauer, C. A., and Andrews, T. B., "The NASA Lewis Research Center Internal Fluid Mechanics Facility," NASA TM 105187, Sept. 1991.

${ }^{6}$ Schubauer, G. B. and Spangenburg, W. G., "Forced Mixing in Boundary Layers," Journal of Fluid Mechanics, Vol. 8, 1960, pp. 10-31.

${ }^{7}$ Wendt, B. J. and Reichert, B. A., "A New Algorithm for Five-Hole Probe Calibration and Data Reduction and its Application to a Rake-Type Probe," Fluid Measurement and Instrumentation Forum - 1993, Vol. 161, edited by R. Gore, T. Morrow, and G. Jones, ASME, 1993, pp. 29 35. 
Public reporting burden for this collection of inlomation is estimated to average 1 hour per response, including the time for reviewing instructions, searching existing data sources, gathering and maintaining the data needed, and completing and revtewing the collection of intormation. Send comments regarding this burden estimate or any other aspect of this (t) Davis Highway, Suite 1204. Adtington. VA 22002-4302, and to the Office of Management and Budget, Papenwork Reduction Project (0704-0188), Washington, DC 20503.

1. AGENCY USE ONLY (Leave blank) 2. REPORT DATE June 1994 3. REPOAT TYPE ANO DATES COVERED Technical Memorandum

\section{TITLE AND SUBTTILE}

5. FUNDING NUMBERS

The Effects of Vortex Ingestion on the Flow in a Diffusing S-Duct

6. AUTHOA(S)

WU-505-62-52

B.J. Wendt and B.A. Reichert

7. PERFORMING ORGANIZATION NAME(S) AND ADDRESS(ES)

8. PERFORMING ORGANIZATION REPORT NUMBER

National Aeronautics and Space Administration

Lewis Research Center

E-8964

Cleveland, Ohio 44135-3191

9. SPONSORING/MONITORING AGENCY NAME(S) AND ADDAESS(ES)

10. SPONSOAINGMONITORING AGENCY REPORT NUMBER

National Aeronautics and Space Administration

Washington, D.C. 20546-0001

NASA TM-106652

AIAA-94-2811

11. SUPPLEMENTARY NOTES

Prepared for the 30th Joint Propulsion Conference cosponsored by AIAA, ASME, SAE, and ASEE, Indianapolis, Indiana, June 27-29, 1994. B.J. Wendt, National Research Council-NASA Research Associate at Lewis Research Center; and B.A. Reichert, NASA Lewis Research Center. Responsible person, B.J. Wendt, organization code 2660, (216) 433-5906.

12a. DISTRIBUTION/AVAILABILITY STATEMENT 12b. DISTRIBUTION CODE

Unclassified -Unlimited

Subject Category 07

13. ABSTRACT (Maximum 200 words)

An experimental study of the effects of an ingested vortex on the flowfield of a diffusing S-duct is reported. The vortex is generated through the use of a stationary pinwheel device mounted upstream of the diffusing S-duct. Three test conditions vary the location of the vortex in the duct inlet crossplane. For each condition of ingested vortex, a baseline S-duct and an S-duct with an array of vortex generators is examined. The data taken consist of duct inlet and exit crossplane surveys of velocity and total pressure. Duct surface flow visualization and static pressure are also recorded. The data acquired in these tests are compared to identical S-duct data taken in the absence of the ingested vortex. The ingested vortex is observed to have a strong influence on the flowfield inside (and exiting) the S-duct, but only when the vortex impinges at the inlet crossplane location coincident with the crossplane location of downstream flow separation within the duct. When the ingested vortex impinges at this location it reduces the extent of flowfield separation inside the baseline duct and promotes stronger crossflow in the exit plane of both the baseline duct and the duct with installed vortex generators. This enhanced crossflow also strengthens the vortices shed from the vortex generators. The other impingement locations of the ingested vortex are found to produce little effect on the flowfield of the duct, with or without vortex generators.

14. SUBJECT TERMS

Inlet flow; Vortex flow; Vortex generators 15. NUMBER OF PAGES 12

16. PRICE CODE $\mathrm{A} 03$

\begin{tabular}{|c|c|}
\hline 17. SECURITY CLASSIFICATION & $\begin{array}{c}\text { 18. SECUATY CLASSIFICATION } \\
\text { OF REPORT } \\
\text { OF THIS PAGE } \\
\text { Unclassified }\end{array}$ \\
Unclassified
\end{tabular}

19. SECURITY CLASSIFICATION OF ABSTRACT Unclassified 